\title{
Three Cases of COVID-19 Variant Delta With and Without Vaccination - Chengdu City, Sichuan Province, April-May, 2021
}

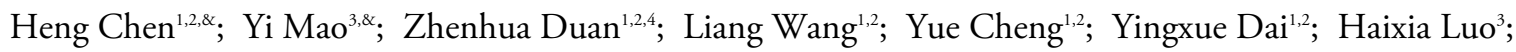 \\ Wenjun $\mathrm{Xie}^{1,2}$; Shuangfeng Fan ${ }^{1,2}$; Yuzhen $\mathrm{Zhou}^{1,2}$; Jingpei $\mathrm{Xu}^{1,2}$; Lan Feng ${ }^{1,2}$; Liwen $\mathrm{Hu}^{1,2}$; \\ Zhu Liu ${ }^{1,2}$; Xian Liang ${ }^{1,2}$; Liangshuang Jiang ${ }^{3, *} ;$ Xiaoli Tuo ${ }^{1,2, *}$
}

On April 26, 2021, a 33-year-old male Chinese sailor returning from India via Kathmandu, Nepal, tested positive for the coronavirus disease 2019 (COVID-19) by Chengdu Customs and was confirmed positive by Chengdu CDC (Case A).

On May 2, 2021, a housewife in the same flight tested positive for COVID-19 by Pengzhou CDC in Sichuan Province during her quarantine period and was confirmed positive by Chengdu CDC the next day (Case B).

On May 9, 2021, a male worker at a cement company in a different flight tested positive for COVID-19 by a third-party testing laboratory during his quarantined period and was confirmed positive by Chengdu CDC the next day (Case C).

Epidemiological investigations revealed that Case A arrived in Kathmandu on April 19 from New Delhi, India and Case B arrived in Kathmandu on April 21 from Uttar Pradesh, India. Case $\mathrm{C}$ had been working in Kathmandu before returning. They all tested negative for polymerase chain reaction (PCR) tests and antibody tests of COVID-19 before boarding.

Case A had been fully vaccinated with two doses of inactivated COVID-19 vaccine (Beijing Institute of Biological Products Co. LTD) with the first dose on January 25 and the second on February 8, 2021. In addition, Case $\mathrm{C}$ was vaccinated (Sinovac Biotech Co. LTD) on October 19 and November 4, 2020. Case B was not vaccinated.

The samples of nasopharyngeal swabs from the three cases were sequenced by Illumina MiniSeq Sequencing platform with commercial kits on April 27 and May 11 , then the whole genome sequences of 29,858, 29,732 , and 29,877 bp by length with depth over 3000X were obtained. Compared to the Wuhan reference sequence (MN908947) (1-2), they shared 20 nucleotide variation sites containing the characteristic spike mutations of T19R, L452R, T478K, D614G,
P681R, and D950N listed in the sub-lineage of B.1.617.2 (variant Delta) that was assigned by a web analytical tool as B.1.617.2 (3), which had been circulating in India since December 2020 and was designated as one of the variants of concern (VOCs) by the World Health Organization (WHO) (4). The phylogenetic tree by CLC Main Workbench 11.0 (QIAGEN, Dusseldorf, Germany) was shown in Figure 1.

Cases A, B, and C of COVID-19 were transferred to the Public Health Clinical Center of Chengdu for treatment in isolation on April 26, May 3, and May 10, 2021, separately (the main clinical events are shown in Figure 2). Soon after the admission, they all were found to have an increase in lung lesions according to chest computed tomography (CT) abnormalities. During the isolation treatment period, 3 differences were found between the vaccinated and unvaccinated patients: 1) The IgGlIgM|total antibodies tests were positive for Case $\mathrm{A}$ on the 4th day, positive for Case $\mathrm{C}$ on the 1st day with high titer, and negative for Case B even on the 7th day (negative for IgM during the entirety of hospitalization and positive for IgG and total antibodies on the 13th day with low titer), which suggested that the time from diagnosis to antibody positivity was shorter in vaccinated cases than in unvaccinated cases [Figure 3 shows the results of IgM and total antibodies results with chemiluminescence immunoassay (CLIA)]. 2) The cycle threshold $(\mathrm{Ct})$ value of fluorescent PCR seems lower in samples from unvaccinated patients than those from the vaccinated ones [Figure 4 shows the results of $\mathrm{Ct}$ values of the open reading frame $(\mathrm{ORF})]$. There is a similar finding that a significant increase in $\mathrm{Ct}$ in vaccinated individuals than matched unvaccinated control group infections $(n=1,888)$ from days 12-37 after vaccination was reported (5). 3) The length of hospitalization was shorter for vaccinated 


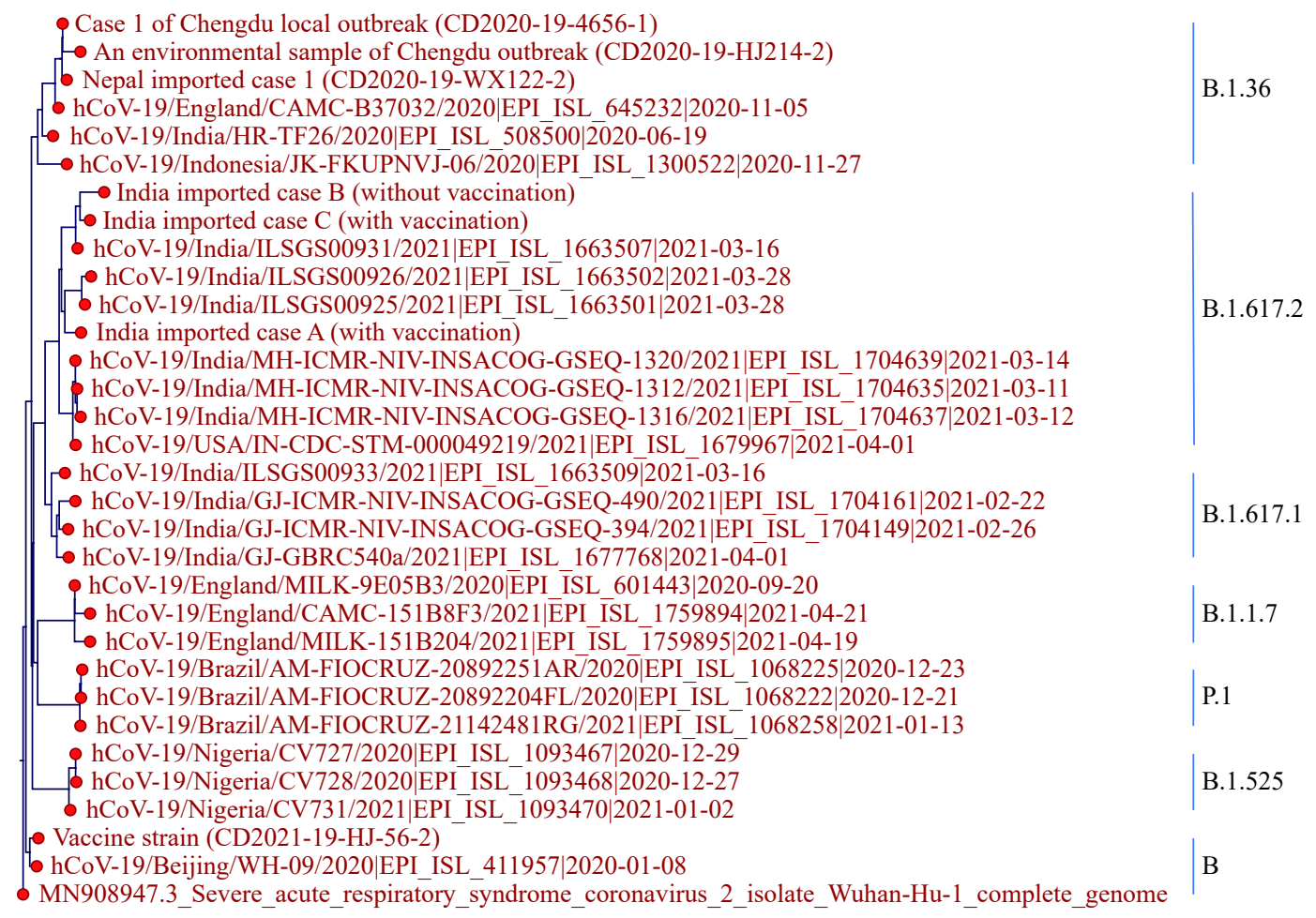

FIGURE 1. Phylogenetic tree based on the full-length genome sequences of the COVID-19 virus.

Note: The main mutations: C241T, C3037T, C14408T, G15451A, C16466T, C21618G, T22917G, C22995A, A23403G, C23604G, G24410A, C25469T, T26767C, T27638C, C27752T, A28461G, G28881T, G29402T, and G29742T.

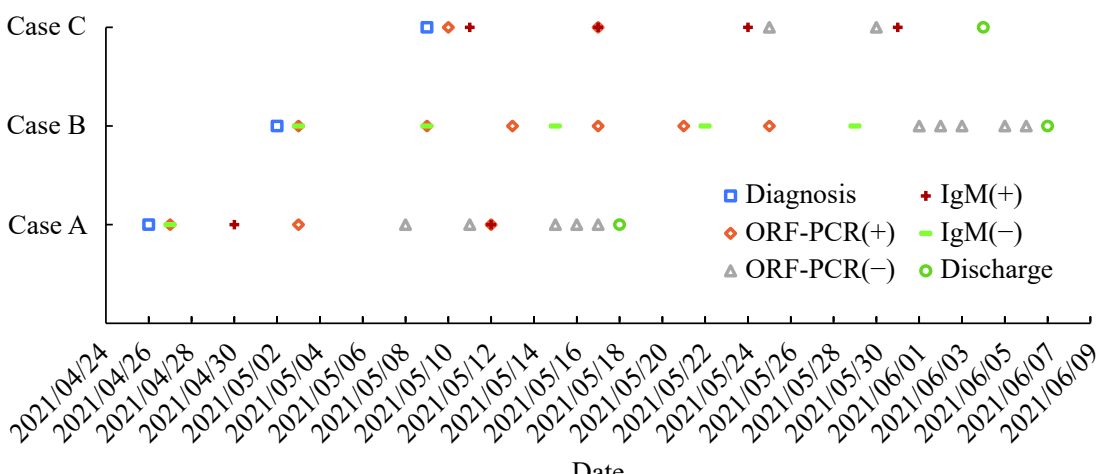

FIGURE 2. The timeline of the main clinical events of the cases.

Note: Cases $A$ and $C$ had been vaccinated while Case $B$ was not vaccinated.

Abbreviations: $\mathrm{ORF}=$ the open reading frame, $\mathrm{PCR}=$ polymerase chain reaction.

patients than for unvaccinated one. Case A and Case C were discharged after 21 and 25 days in the hospital, respectively, and Case B was discharged on June 7 after 36 days.

The variant Delta strains were imported into China and exerted a great threat to the prevention and control of COVID-19. Further studies looking into the epidemiological impacts of the variants and the effects of the vaccine are urgently needed. As local outbreaks of COVID-19 are still reported in Anhui, Liaoning, and Guangdong, vaccination should be taken as the first strategy and promoted efficiently for the disease control for COVID-19.

Acknowledgements: Participating CDC colleagues.

Funding: Non-Profit Central Research Institute Fund of Chinese Academy of Medical Sciences (2020PT330-005).

doi: $10.46234 / \mathrm{ccdcw} 2021.137$

\# Corresponding authors: Liangshuang Jiang, 502830704@qq.com; Xiaoli Tuo,3439682@qq.com. 


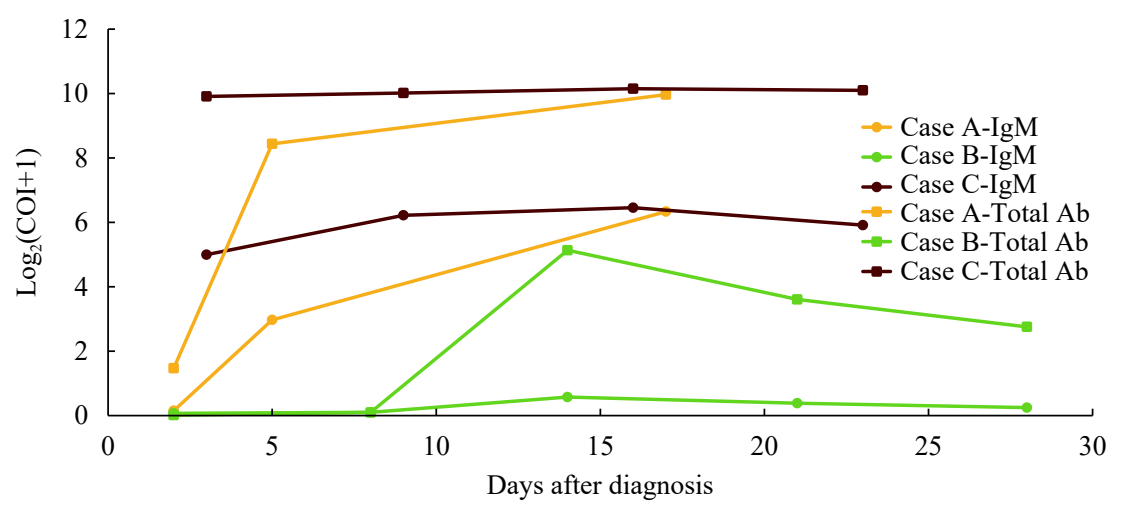

FIGURE 3. The antibody titer of IgM and total antibodies analyzed using chemiluminescence analysis (CLIA) during the isolation treatment period.

Note: Cases $A$ and $C$ had been vaccinated while Case B was not vaccinated. All cases were negative for IgM with ELISA on Day 1 , which is not shown in this figure.

Abbreviations: Total $\mathrm{Ab}=$ total antibodies, $\mathrm{COI}=$ cut off index.

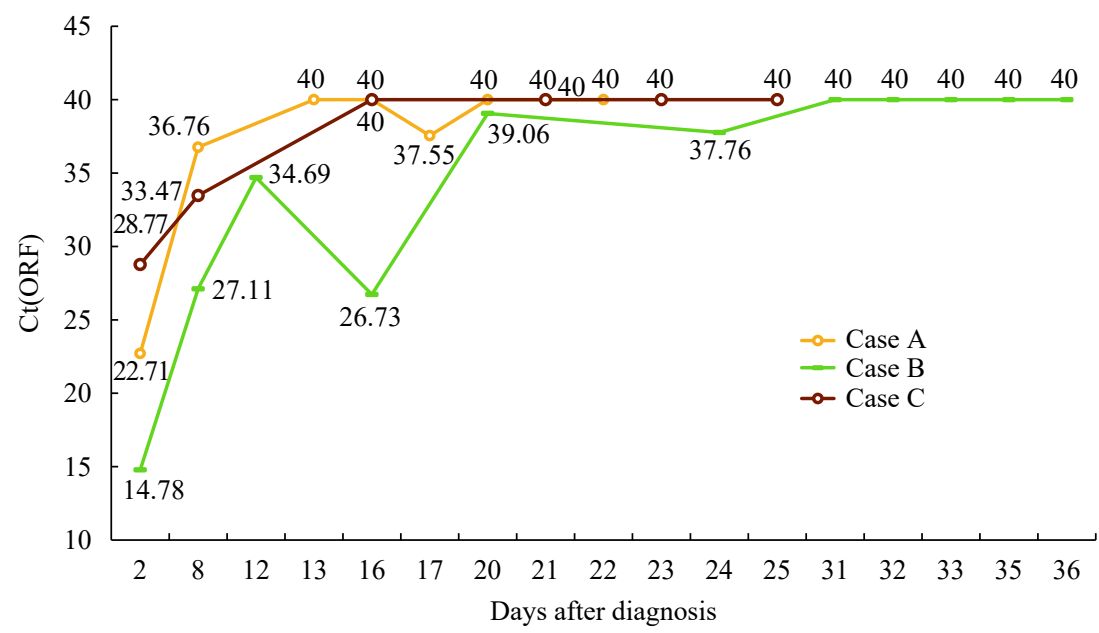

FIGURE 4. The CT values of ORF with PCR during the isolation treatment period.

Note: Cases A and C had been vaccinated while Case B was not vaccinated. A CT value of 40 was used to assume a negative result.

Abbreviations: $\mathrm{CT}=$ computed tomography; $\mathrm{ORF}=$ open reading frame; $\mathrm{PCR}=$ polymerase chain reaction.

\footnotetext{
${ }^{1}$ Chengdu Workstation for Emerging Infectious Disease Control and Prevention, Chinese Academy of Medical Sciences, Chengdu, Sichuan, China; ${ }^{2}$ Chengdu Center for Disease Control and Prevention, Chengdu, Sichuan, China; ${ }^{3}$ Public Health Clinical Center of Chengdu, Chengdu, Sichuan, China; ${ }^{4}$ Chinese Field Epidemiology Training Program, Chinese Center for Disease Control and Prevention, Beijing, China.

\& Joint first authors.
}

Submitted: June 09, 2021; Accepted: June 10, 2021

\section{REFERENCES}

1. GenBank. Severe acute respiratory syndrome coronavirus 2 isolate
Wuhan-Hu-1, complete genome. https://www.ncbi.nlm.nih.gov/nuccore/ MN908947. [2021-5-26].

2. Wu F, Zhao S, Yu B, Chen YM, Wang W, Song ZG, et al. A new coronavirus associated with human respiratory disease in China. Nature 2020;579(7798):265 - 9. http://dx.doi.org/10.1038/s41586-020-2008-3.

3. Pangolin COVID-19 Lineage Assigner. Phylogenetic assignment of named global outbreak LINeages. https://pangolin.cog-uk.io/. [2021-526].

4. WHO Weekly epidemiological update on COVID-19 - 8 June 2021. https://www.who.int/publications/m/item/weekly-epidemiologicalupdate-on-covid-19---8-june-2021. [2021-6-8].

5. Levine-Tiefenbrun M, Yelin I, Katz R, Herzel E, Golan Z, Schreiber L, et al. Initial report of decreased SARS-CoV-2 viral load after inoculation with the BNT162b2 vaccine. Nat Med 2021;27(5):790 - 2. http://dx. doi.org/10.1038/s41591-021-01316-7. 